\title{
PRODUTIVIDADE DE SORGO EM PLANTIO DIRETO NO SUDOESTE GOIANO COM DIFERENTES DOSES DE FERTILIZANTE ORGANOMINERAL
}

Joaquim Júlio de Almeida Júnior ${ }^{1}$, Adriano Bernardo Leal ${ }^{2}$, Beatriz Campos Miranda ${ }^{3}$, Renan Costa Barros ${ }^{4}$, Geovana Almeida Carmo ${ }^{5}$

1 Pós doutorando, UC - Universidade de Coimbra, joaquimjuliojr@gmail.com. Coimbra, Portugal.

2 Acadêmico do curso de Engenharia Agronômica. UniFimes-Centro Universitário de Mineiros. Goiás. Brasil.

3 Acadêmica do curso de Engenharia Florestal. UniFimes-Centro Universitário de Mineiros. Goiás. Brasil.

4 Acadêmico do curso de Engenharia Agronômica. UniFimes-Centro Universitário de Mineiros. Goiás. Brasil.

5 Acadêmica do curso de Engenharia Agronômica, UniCerrado-Centro Universitário de Goiatuba. Goiás, Brasil.

Recebido em: 06/04/2018 - Aprovado em: 10/06/2018 - Publicado em: 20/06/2018 DOI: 10.18677/EnciBio_2018A23

\section{RESUMO}

A cultura do sorgo (Sorghum bicolor (L.) Moench) tem uma ampla diversidade em aspectos fisiológicos e produtivos, tendo características ideais para semeaduras em regiões de baixa precipitação ou safrinha. O presente estudo teve como objetivo testar a influência de diferentes doses de fertilizante organomineral na cultura do sorgo granífero Enforcer, avaliar as características agronômicas da planta e produtividade em sacas por hectare, no sistema de plantio direto (SPD). O trabalho foi desenvolvido na área experimental do Núcleo de Estudo e Pesquisa em Fitotecnia - MINEIROS (GO), ano agrícola 2017. Os tratamentos se constituíram em T1: 0,0 Kg ha-1; T2: $150 \mathrm{Kg} \mathrm{ha}^{-1}$ (A.O.M.); T3: $300 \mathrm{Kg} \mathrm{ha}^{-1}$ (A.O.M.); T4: $450 \mathrm{Kg} \mathrm{ha-}$ ${ }^{1}$ (A.O.M.); T5: $600 \mathrm{Kg} \mathrm{ha}^{-1}$; T6: $750 \mathrm{Kg} \mathrm{ha}^{-1}$ (A.O.M.); T7: $900 \mathrm{Kg} \mathrm{ha}^{-1}$ (A.O.M.) com a utilização da cultivar de sorgo Enforcer. O delineamento experimental foi em blocos casualizados, esquema $7 \times 1$ com quatro repetições. Os dados foram analisados pelo programa Assistat e submetidos à análise de variância, sendo as médias comparadas pelo teste $t$ a $5 \%$ de probabilidade. O fertilizante organomineral usado no sorgo Enforcer promoveu ganho de produtividade para cultura em diversas doses em comparação com o tratamento controle dose 0,0 no sistema de plantio direto. Também pôde-se afirmar que é uma alternativa de fertilização para as culturas com grande apelo agroecológico.

PALAVRAS-CHAVE: Desempenho produtivo. Eficiência. Material orgânico. Nutrição Agroecologia. Sustentabilidade.

PRODUCTIVE CHARACTERISTICS OF SORGHUM ENFORCER IN A DIRECT PLANTAIN SYSTEM IN THE SOUTHWEST REGION OF GOIANO, USING DIFFERENT DOSES OF ORGANOMINERAL FETILIZANTES 


\begin{abstract}
The sorghum (Sorghum bicolor (L.) Moench) culture has a wide diversity in physiological and productive aspects, having ideal characteristics for sowing in regions with low rainfall or low rainfall. The present study had as objective to test the influence of different doses of organomineral fertilizer on the grain sorghum Enforcer crop and to evaluate the agronomic characteristics of the plant and yield in sacks per hectare in the no - tillage system (SPD). The work was carried out in the experimental area of the Nucleus of Study and Research in Plant Science MINEIROS (GO.), In the agricultural year 2017. The treatments consisted of T1: 0,0 $\mathrm{Kg} \mathrm{ha}^{-1}$; T2: $150 \mathrm{~kg} \mathrm{ha}{ }^{-1}$ (A.O.M.); T3: $300 \mathrm{~kg} \mathrm{ha}^{-1}$ (A.O.M.); T4: $450 \mathrm{~kg} \mathrm{ha}^{-1}$ (A.O.M.); T5: $600 \mathrm{~kg} \mathrm{ha}^{-1}$; T6: $750 \mathrm{~kg} \mathrm{ha}^{-1}$ (A.O.M.); T7: $900 \mathrm{~kg} \mathrm{ha}^{-1}$ (A.O.M.). With the use of the sorghum cultivar Enforcer. The experimental design was a randomized complete block, $7 \times 1$ scheme with four replications, the data were analyzed by the Assistat program and submitted to analysis of variance, the means being compared by the $t$ test. The organomineral fertilizer used in the Enforcer sorghum promoted a productivity gain for cultivation in several doses compared to the 0,0 dose control treatment in the no-tillage system. It can also be said that it is a fertilization alternative for crops with great agroecological appeal.
\end{abstract}

KEYWORDS: Productive performance. Efficiency. Organic material. Nutrition Agroecology. Sustainability.

\title{
INTRODUÇÃO
}

No Brasil o Sistema de plantio direto (SPD) foi iniciado da década de 70, na região Sul, com o objetivo de minimizar impactos causados pelo plantio convencional, aliado a vários fatores que impactaram na estrutura, microfauna dos solos e principalmente a fertilidade. A utilização intensiva como gradagens torna o solo mais exposto e consequentemente com maior susceptibilidade a erosões, causando assim uma queda enorme em nutrição destas áreas produtivas, estes aspectos ligados a presença de palhada nestas regiões podem favorecer podem favorecer a disponibilidade de nutrientes solúveis na solução do solo, isso deve-se ao fato da característica de retenção de água e umidade que a matéria orgânica possibilita ao solo (SILVA et al. 2015).

Carvalho et al., (2011) afirmam que a cultura do sorgo é uma opção altamente viável para cultivar em segunda safra na região do sudoeste goiano por possuir rusticidade e assim atendendo aptidão da região que possui características quente e com pouca disponibilidade de água. Esta cultura apresenta manejo simples, similar ao milho, com vantagens como tolerância à seca, período de plantio mais amplo e lavoura totalmente mecanizável, com múltiplas aplicações com um ótimo incremento nas adubações organomineral, podendo até superar outras culturas em produtividades de grãos (CARVALHO et al. 2011).

Este vegetal vem se expandindo significativamente nos últimos anos consolidando como cultura rentável na época de segunda safra, conhecida como "safrinha". Com vários fatores que auxiliam em seu potencial produtivo, a planta de sorgo possui características fisiológicas que permitem paralisar seu crescimento ou diminuir o seu metabolismo durante o estresse hídrico, e reiniciá-lo quando a água se tornar disponível (LOURENÇÃO; BAGEGA. 2012). 
Para alcançar produções de grãos satisfatórias que atendam a demanda é necessário o emprego de práticas de cultivos agrícolas adequados. Também é indispensável à realização de adubações respeitando a recomendação de análise de solo, de modo que a cultura desenvolva em ambiente equilibrado nutricionalmente (ALMEIDA JÚNIOR et al., 2016).

O uso de fertilizante organomineral corresponde a uma solução tecnológica, tanto sob o ponto de vista ambiental, como agronômico, pois combinam minerais fertilizantes minerais (matéria prima minerais) e material orgânico (resíduos orgânicos). Desta forma, o uso deste fertilizante pode ser uma alternativa inovadora na produção de grãos, pois pode diminuir os custos de produção, otimizar recursos naturais que não poderiam ser descartados e ainda gerar economia (COSTA ,2017).

Posto isto, o presente estudo teve por objetivo testar a influência de diferentes doses de fertilizante organomineral na cultura do sorgo granífero Enforcer e avaliar as características agronômica da planta e produtividade em sacas por hectare no sistema de plantio direto.

\section{MATERIAL E MÉTODOS}

O projeto foi conduzido no ano agrícola de 2017 na área experimental do Núcleo de Ensino e Pesquisa em Fitotecnia - MINEIROS/GO, apresentando como coordenadas geográficas aproximadas, $17^{\circ} 58^{\prime} \mathrm{S}$ de latitude e $45^{\circ} 22^{\prime} \mathrm{W}$ de longitude e com $845 \mathrm{~m}$ de altitude. O clima predominante da região, conforme classificação de Köppen (2013) é do tipo Aw, definido como tropical úmido com estação chuvosa no verão e seca no inverno. A precipitação pluvial média anual é de $1.830 \mathrm{~mm}$, com temperatura média anual de aproximadamente $25^{\circ} \mathrm{C}$ e umidade relativa do ar média anual de $66 \%$.

O período chuvoso se estende de outubro a março, sendo que os meses de dezembro, janeiro e fevereiro constituem o trimestre com maior precipitação, e o trimestre mais seco corresponde aos meses de junho, julho e agosto (média de 27 $\mathrm{mm})$.

O solo predominante da área, conforme a nova denominação do Sistema Brasileiro de Classificação de Solos como é o Neossolo Quartzarênico de textura arenosa (EMBRAPA, 2013), o qual foi originalmente ocupado por vegetação de Cerrado e vem sendo explorado por culturas anuais a mais de 15 anos (Tabela 1).

TABELA 1. Resultados obtidos na análise química do solo, coletada na área experimental do Núcleo de Ensino e Pesquisa em Fitotecnia da UNIFIMES, amostrada antes do plantio do sorgo safrinha BRS 380 . Município de Mineiro/GO. 2017.

\begin{tabular}{|c|c|c|c|c|c|c|c|c|c|c|c|}
\hline Profundida & $\mathrm{pH}$ & $\mathrm{P}$ (Mel) & $\mathrm{K}^{+}$ & $\mathrm{Ca}$ & $\mathrm{Mg}$ & $\overline{\mathrm{Al}}$ & $\mathrm{H}+\mathrm{Al}$ & S.B. & CTC & $\mathrm{V}$ & M.O. \\
\hline & $\mathrm{CaCl}_{2}$ & $\mathrm{mg} \mathrm{dm}^{-}$ & \multicolumn{7}{|c|}{ mmolc $\mathrm{dm}^{-3}$} & $\%$ & $\mathrm{gdm}^{-3}$ \\
\hline $0-20$ & 4,9 & 7 & 1,6 & 18 & 10 & 0 & 31 & 29,8 & 60,8 & 49,05 & 22 \\
\hline $20-40$ & 4,9 & 61 & 1 & 5 & 3 & 0 & 29 & 9 & 38 & 23,76 & 18 \\
\hline
\end{tabular}


Fonte: Os Autores, (2017).

Os atributos químicos do solo $(\mathrm{pH}, \mathrm{K}, \mathrm{Ca}, \mathrm{Mg}, \mathrm{H}+\mathrm{Al}$ e $\mathrm{Al})$ foram determinados, nas camadas de 0,0-0,20 m e 0,20-0,40 m, no Laboratório de Fertilidade do Solo da instituição. Esses atributos do solo foram avaliados antes da implantação do projeto de pesquisa para conhecer as características químicas da área experimental, segundo a metodologia proposta por (RAIJ ;QUAGGIO. 1983),

Os tratamentos foram constituídos em T1: $0,0 \mathrm{Kg}^{-1} ; \mathrm{T} 2: 150 \mathrm{Kg} \mathrm{ha}{ }^{-1}$ (A.O.M.); T3: $300 \mathrm{Kg} \mathrm{ha}^{-1}$ (A.O.M.); T4: $450 \mathrm{Kg} \mathrm{ha}^{-1}$ (A.O.M.); T5: $600 \mathrm{Kg} \mathrm{ha}^{-1}$; T6: 750 $\mathrm{Kg} \mathrm{ha}^{-1}$ (A.O.M.); T7: $900 \mathrm{Kg} \mathrm{ha}^{-1}$ (A.O.M.). A cultivar de sorgo granífero Enforcer, foi avaliada as características agronômicas da planta como altura de planta, população de planta, peso de mil grãos e produtividade em sacas por hectare.

O delineamento experimental foi em blocos casualizados, esquema $7 \times 1 \mathrm{com}$ 4 repetições. Cada parcela experimental foi constituída de 4 linhas de 2,0 metros de comprimento e espaçamento de 0,5 metros ocupou uma área total de $2,0 \mathrm{~m}^{2}(2,0 \mathrm{~m}$ $\times 0,5 \mathrm{~m} \times 2,0$ ). Os dados foram analisados pelo programa Assistat ${ }^{\circledR}$ (SILVA ; AZEVEDO, 2016). Os dados obtidos foram submetidos à análise de variância, sendo as médias comparadas pelo teste $t$, quando detectada significância para a ANOVA a $5 \%$ de probabilidade para a comparação entre médias.

\section{RESULTADOS E DISCUSSÃO}

Os dados de altura de plantas, população de plantas, produtividade em sacas por hectare e peso de 1000 grãos apresentaram coeficiente de variação (CV) satisfatórios. Estes dados foram obtidos com precisão conforme classificação proposta por Carvalho et al. (2003). Os resultados encontrados neste trabalho coincidem com os encontrado por Carvalho (2011) e Nakayama et al (2013), em que os CV se encontraram dentro da faixa considerados médias, apresentando baixa dispersão.

Visualiza-se na Tabela 2 que nos tratamentos os dados das variáveis tecnológicas testadas, altura de plantas, população de plantas, produtividade sacas por hectare e peso de mil grãos não ocorreram significância entre as variáveis testadas. No fator de variação bloco as variáveis tecnológicas testadas somente altura de planta foi significativa. Para população de plantas, produtividade sacas por hectare e peso de 1000 grãos não foi detectado significância em nenhuma delas.

TABELA 2. Resumo da análise de variância das características agronômicas do sorgo Enforcer em função das dores crescente do fertilizante organomineral 04-14-08, 2017. AP: Altura de plantas; PP: População de Plantas; PScha-1 ${ }^{-1}$ Produtividade sacas por hectare; PMG: Peso de mil grãos.

\begin{tabular}{cccccc}
\hline FV & GL & AP & PP & P $($ Sc $) h^{-1}$ & PMG \\
\hline Blocos & 3 & $4,7154^{*}$ & $2,5103^{\text {ns }}$ & $1,9015^{\text {ns }}$ & $2,9468^{\text {ns }}$ \\
Tratamentos & 6 & $0,5455^{\text {ns }}$ & $0,8835^{\text {ns }}$ & $1,8028^{\text {ns }}$ & $2,6101^{\text {ns }}$ \\
Resíduo & 18 & - & - & - & - \\
\hline DMS & - & 0.09 & 2.93 & 76.27 & 5.40 \\
\hline CV $(\%)$ & - & 5.68 & 32.99 & 32.91 & 10.41 \\
\hline
\end{tabular}


Os símbolos "** e *" corresponde ao nível de significância sendo: **significativo ao nível de $1 \%$ de probabilidade; * significativo ao nível de $5 \%$ de probabilidade; ns: não significativo.

Fonte: Os Autores, (2017).

Percebe-se na Tabela 3 que produtividade em sacas por hectare ocorreu diferença estatística significativa entre os tratamentos utilizados, em que os melhores resultados foram obtidos através dos tratamentos T5 com a dose de 600 $\mathrm{Kg} \mathrm{ha}^{-1}$, T6 com a dose de $750 \mathrm{Kg} \mathrm{ha}^{-1}$, T2 com a dose de $150 \mathrm{Kg} \mathrm{ha}^{-1}$, T4 com a dose de $450 \mathrm{Kg} \mathrm{ha}^{-1}$ e T3 com a dose $300 \mathrm{Kg} \mathrm{ha}^{-1}$, já os tratamentos que ficaram com valores inferiores entre os demais foram os tratamentos T1 com a dose $0,0 \mathrm{Kg} \mathrm{ha}^{-1}$, T3 com a dose $300 \mathrm{Kg} \mathrm{ha}^{-1}$, T7 com a dose de $900 \mathrm{Kg} \mathrm{ha}^{-1}$, T4 com a dose de 450 $\mathrm{Kg} \mathrm{ha}^{-1}$ e T5 com a dose de $600 \mathrm{Kg} \mathrm{ha}^{-1}$. É possível afirmar que mesmo com uma boa fertilidade de solo o fertilizante organomineral utilizado promoveu um ganho significativo na produtividade em sacas por hectare entre os tratamentos utilizados. Em trabalho realizado por Ciancio (2010) com sorgo, milho e feijão, foi encontrado efeito significativo na produtividade com utilização de fertilizante orgânico (cama de peru). Em trabalho com milho não foi observada diferença significativa quanto as diferentes doses e combinações de fertilizantes orgânicos e químicos (SILVA et al., 2015).

TABELA 3. Médias das variáveis tecnológicas da cultura de sorgo, cultivar Enforcer. Em função das doses crescente do fertilizante organomineral 04-14-08. Mineiros estado de Goiás, 2017. AP: Altura de plantas; PP: População de Plantas; $\mathrm{P}(\mathrm{Sc})$ ha $^{-1}$ : Produtividade sacas por hectare; PMG: Peso de mil grãos.

\begin{tabular}{cccccc}
\hline Tratamentos & Dose $\left(\mathrm{Kg} \mathrm{ha}^{-1}\right)$ & $\mathrm{AP}(\mathrm{m})$ & $\mathrm{PP}$ & $\mathrm{P}(\mathrm{Sc})$ ha $^{-1}$ & PMG $(\mathrm{g})$ \\
\hline T1 & 0,0 & 1.06 & 6,41 & $97.50 \mathrm{~b}$ & $33.95 \mathrm{ab}$ \\
T2 & 150 & 1.09 & 5,16 & $191.81 \mathrm{a}$ & $35.23 \mathrm{a}$ \\
T3 & 300 & 1.06 & 5,00 & $130.85 \mathrm{ab}$ & $33.72 \mathrm{ab}$ \\
T4 & 450 & 1.10 & 6,91 & $155.03 \mathrm{ab}$ & $29.55 \mathrm{~b}$ \\
T5 & 600 & 1.03 & 6,0 & $167.06 \mathrm{ab}$ & $36.93 \mathrm{a}$ \\
T6 & 750 & 1.06 & 5,16 & $196.84 \mathrm{a}$ & $36.74 \mathrm{a}$ \\
T7 & 900 & 1.07 & 7,33 & $153.35 \mathrm{ab}$ & $38.55 \mathrm{a}$ \\
\hline DMS & - & 0.09 & 2.93 & 76.27 & 5.40 \\
\hline CV \% & - & 5.68 & 32.99 & 32.91 & 10.41 \\
\hline
\end{tabular}

As médias seguidas pelas mesmas letras minúscula na coluna não diferem estatisticamente entre si pelo teste t ao nível de $5 \%$ de probabilidade.

Fonte: Os Autores, (2017).

A Tabela 3 mostra diferença estatística significativa entre os tratamentos utilizados para a variável tecnológica de peso de mil grãos, onde os que demonstraram os melhores resultados foram os tratamentos T7 com a dose de 900 $\mathrm{Kg} \mathrm{ha}^{-1}$, T5 com a dose de $600 \mathrm{Kg} \mathrm{ha}^{-1}$, T6 com a dose de $750 \mathrm{Kg} \mathrm{ha}^{-1}$, T1 com a dose $0,0 \mathrm{Kg} \mathrm{ha}^{-1}$, T3 com a dose de $300 \mathrm{Kg} \mathrm{ha}^{-1}$, T2 com a dose de $150 \mathrm{Kg} \mathrm{ha}^{-1} \mathrm{e}$ os tratamentos que obtiveram valores estatisticamente inferiores aos demais foram T4 com a dose de $450 \mathrm{Kg} \mathrm{ha}^{-1}$, T1 com a dose $0,0 \mathrm{Kg} \mathrm{ha}^{-1}$ e T3 com a dose de $300 \mathrm{Kg}$ ha $^{-1}$. Sarto et al. (2010) afirmaram que em solo arenoso, a aplicação de fertilizante 
organomineral proporcionou incremento até a dose de $140 \mathrm{~kg} \mathrm{ha}^{-1}$ com produção máxima de $26 \mathrm{~g}$ vaso $^{-1}$.

Foi possível observar na Figura 1 que a curva polinomial para variável tecnologica produtividade em sacas por hectare, ao ocorrer o acréscimo na dose do fertilizante organomineral, houve a elevação da produtividade em sacas por hectare ate a dose de $750 \mathrm{Kg} \mathrm{ha}^{-1}$, registrado no tratamento T6, decrescendo a produtividade em sacas por hectare para o tratamento T7 com a dose de $900 \mathrm{Kg} \mathrm{ha}^{-1}$. Pode-se surgerir que ocorreu toxicidade na planta ou saturação de nutriente pela planta. $O$ incremento da produtividade de grãos em função das doses de dejeto líquido de suíno e esterco de peru na ausência e presença da adubação mineral, está relacionada à aplicação de um dejeto com alto teor de matéria seca, o que significou uma maior adição de $\mathrm{N}$ ao solo, além dos macronutrientes $\mathrm{P}, \mathrm{K}, \mathrm{Ca}$ e $\mathrm{Mg}$ (CIANCIO, 2010).

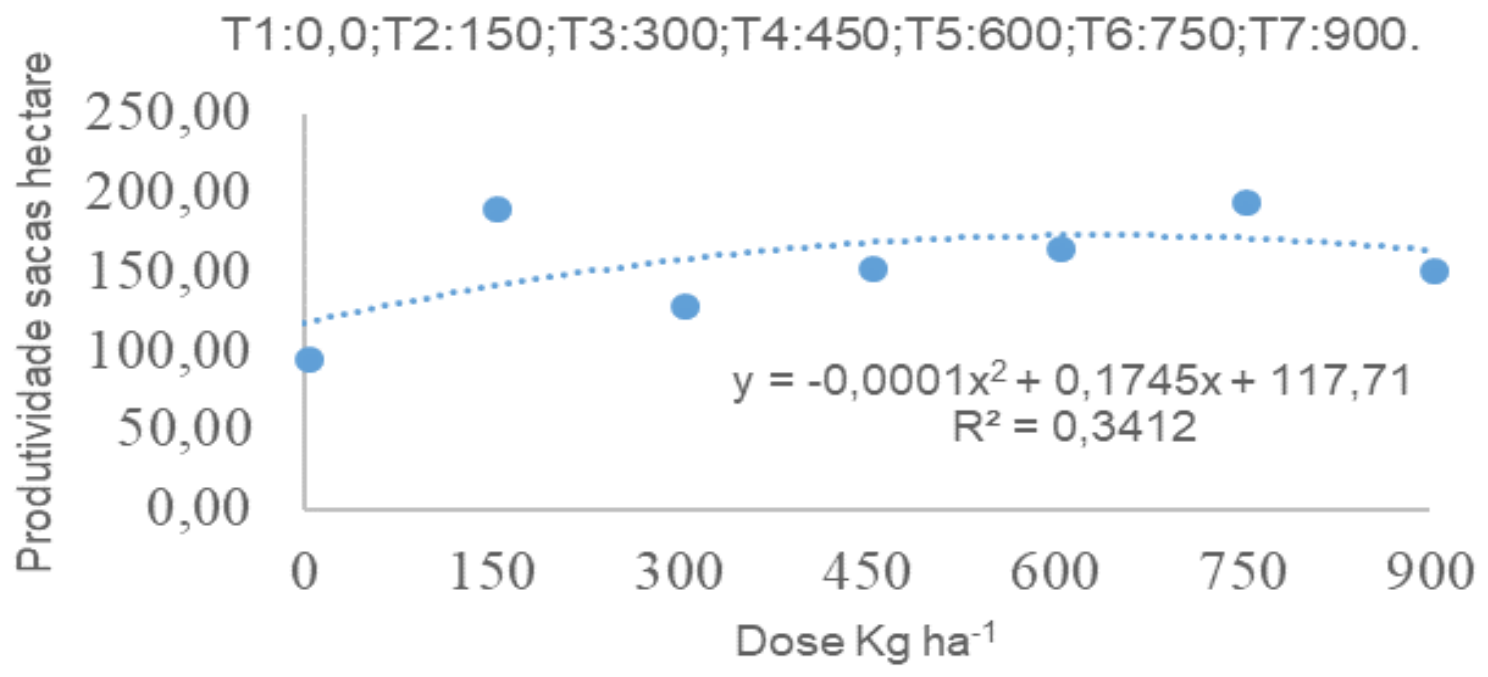

FIGURA 1. Curva polinomial para variável tecnologica produtividade em sacas por hectare da cultura de sorgo, cultivar Enforcer, Em função das doses crescente do fertilizante organomineral 04-14-08.

Fonte: Os Autores, (2017).

Nota-se na Figura 2 que a curva polinomial para variável tecnologica peso de mil grãos, foi ascendente, sendo o tratamento $\mathrm{T} 7 \mathrm{com}$ a dose de $900 \mathrm{Kg} \mathrm{ha}^{-1} \mathrm{o}$ que obteve o melhor resultado em peso de mil grãos, equiparando aos demais tratamentos, com excessão do tratamento T4, tratamento controle com dose $450 \mathrm{Kg}$ ha $^{-1}$ de fertilizante organomineral utilizado obtendo o menor peso de mil grãos em relação aos demais tratamentos. Sarto et al. (2010) trabalharam com sorgo granífero utilizando fertilizante organomineral em solo argiloso e registraram que ocorreu incremento da produção de matéria seca, resultado semelhante ao encontrado neste estudo. 
T1:0,0;T2:150;T3:300;T4:450;T5:600;T6:750;T7:900.

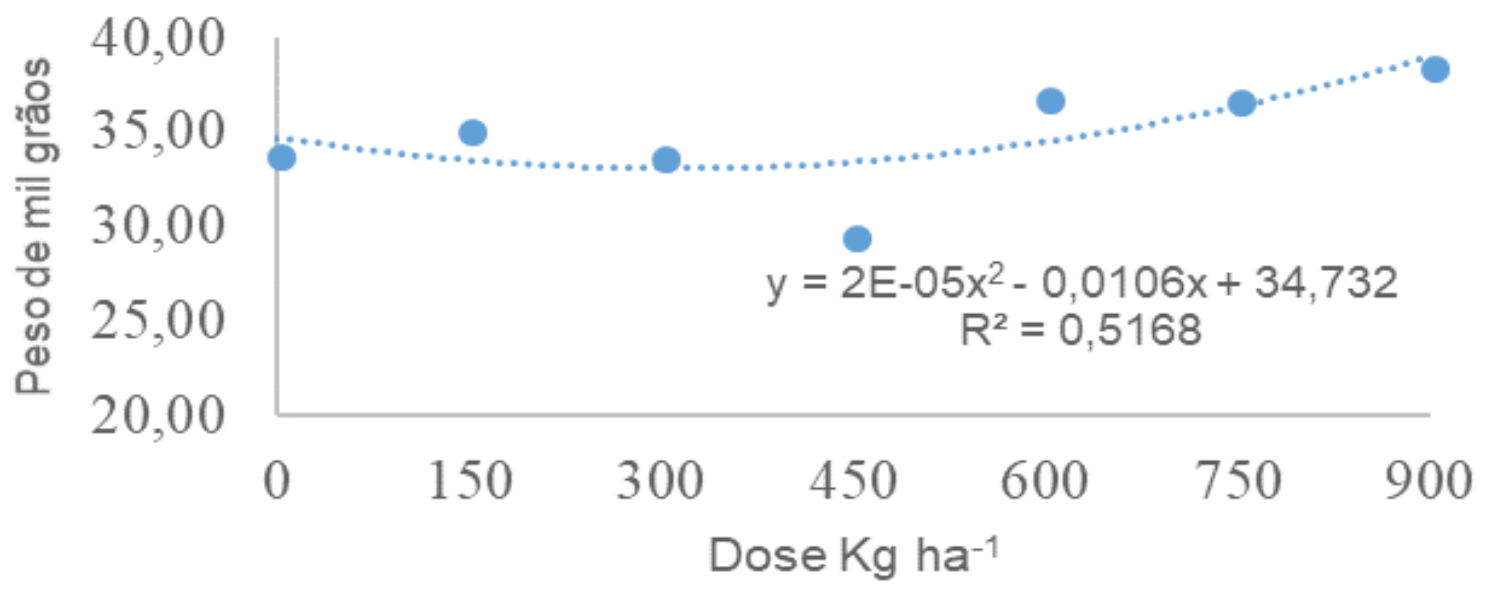

FIGURA 2. Curva polinomial para variável tecnologica peso de mil grãos da cultura de sorgo, cultivar Enforcer. Em função das doses crescente do fertilizante organomineral 04-14-08.

Fonte: Os Autores, (2017).

\section{CONCLUSÃO}

O fertilizante organomineral usado no sorgo granífero Enforcer, promoveu ganho de produtividade em diversas doses em comparação com o tratamento controle no sistema de plantio direto.

Quando comparamos o T1 (controle) com o tratamento de maior dose utilizada T7, podemos observar que o melhor resultado foi obtido no T6, podendo ser em função de excesso nutricional.

Pode-se afirmar que o fertilizante organomineral é uma alternativa de fertilização para as culturas com grande apelo agroecológico.

\section{AGRADECIMENTOS}

A Empresa Ferticel Indústria de Fertilizantes Ltda por contribuir com informações técnicas e fertilizante organomineral utilizado neste projeto. A todos os acadêmicos do curso de Engenharia Agronômica pela participação no desenvolvimento deste projeto.

\section{REFERÊNCIAS}

ALMEIDA JÚNIOR, J. J; SMILJANIC, K. B. A; MATOS; F. S. A; JUSTINO, P. R. V; SILVA, W. T. R; CREMONESE, H. S. Utilização de Adubação Organomineral na Cultura da Soja. II Colóquio Estadual e Pesquisa Multidisciplinar, 2016. ISSN 2527-2500.

CARVALHO, E. R; REZENDE, P. M; ANDRADE, M. J. B; ALEXANDRE MARTINS PASSOS, A; OLIVEIRA, J. A. Fertilizante mineral e resíduo orgânico sobre 
características agronômicas da soja e nutrientes no solo. Revista Ciência Agronômica, v. 42, n. 4, p. 930-939, out-dez, 2011.

CIANCIO, N. H. R; Produção de grãos, matéria seca e acúmulo de nutrientes em culturas submetidas à adubação orgânica e mineral. Santa Maria, 2010. 85 f. Dissertação. Universidade Federal de Santa Maria. Centro de Ciências Rurais, Programa de Pós-Graduação em Ciência do Solo, 2010.

COSTA, F. K. D; Desempenho agronômico da soja convencional cultivada com fertilizantes organomineral e mineral. Dissertação. UniRV - Universidade de Rio Verde. Programa de Pós-Graduação em Produção Vegetal. 2017.

EMBRAPA - Empresa Brasileira de Pesquisa Agropecuária. Sistema Brasileiro de Classificação de Solos. Brasília, $3^{a}$ edição, 2013. 353 p.

KÖPPEN, G; ALVARES, C.A; STAPE, J.L; SENTELHAS, P.C; DE GONÇALVES, M; LEONARDO, J; GERD, S; Köppen's Climate Classification Map for Brazil. (em inglês). Meteorologische Zeitschrift. p. 711-728, 2013.

LOURENÇÃO, A. L. F; BAGEGA, D; Tecnologias para a cultura do sorgo. Fundação M.S. Boletim informativo 11. p.138-141. 2012.

NAKAYAMA, F. T.; PINHEIRO, G. A. S.; ZERBINI, E. F. Eficiência do fertilizante organomineral na produtividade do feijoeiro (Phaseolus vulgaris L.) em sistema de semeadura direta. IX Fórum Ambiental da Alta Paulista. Periódico Eletrônico v.9, n.7, p. 122-138, 2013. ISSN 1980-0827. DOI: 10.17271/19800827.

RAIJ, B. V; QUAGGIO, J.A. Métodos de Análise de Solo para Fins de Fertilidade. Campinas, Instituto Agronômico, 1983. 31p. (Boletim técnico, 81).

SARTO, M. V. M; STEINER, F; PIVETTA, L. A; CASTOLDI, G; LÁZARO, R. L; Crescimento do Sorgo Granífero em Função da Adubação Organomineral e Química em Solos de Diferente Textura. In: XXVIII Congresso Nacional de Milho e Sorgo. 2010.

SILVA, F. A. S; AZEVEDO, C.A.V. The Assistat Software Version 7.7 and its use in the analysis of experimental. Agri \& Aquaculture Journals. v. 11, n.39, p.3733-3740, 2016.

SILVA, P. C; SILVA, K. R; COSTA, R. A; NEVES, P. M; FARIAS, L. S; MARTINS, D. $A$; Adubos orgânicos no desenvolvimento vegetativo e produtividade da cultura do milho. In: XXXV Congresso Brasileiro de Ciência do Solo. Centro de Convenções, Natal-RN. 2015. 